\title{
Interactive Multidimensional Scaling of Cognitive Structure Underlying Person Perception
}

\author{
Jerard Kehoe \\ Virginia Polytechnic Institute and State University \\ Thomas J. Reynolds \\ University of Southern California
}

\begin{abstract}
A computer-interactive multidimensional scaling (MDS) program (INTERSCAL) was used together with free response methods to represent and label dimensions of individual cognitive structure underlying person perception. INTERSCAL reduced by $40 \%$ the number of judgments required by each respondent over traditional complete judgment MDS methods. The dimensional structures derived by INTERSCAL were predictive of semantic differential type judgments, Repertory Grid Test triad judgments, and independent pair-comparison judgments. Typically, one or two dimensions were recovered and were labeled evaluative and potency dimensions, respectively. These dimensional structures were stable within individuals over a period of ten weeks. This pattern of overall consistency implies that particular characteristics of an individ. ual's structure and changes in the relative location of the stimuli over time may be given serious consideration, and that INTERSCAL is an efficient method for scaling such dimensional structures.
\end{abstract}

A person may be thought of as bringing to a situation a cognitive structure which gives organization and meaning to information obtained through perceptions of objects and events. The cognitive structure underlying person perception may be thought of as the processes mediating the perception of the relation-

APPLIED PSYCHOLOGICAL MEASUREMENT

Vol. 1. No. 2 Spring 1977 pp. 155-169

(c) Copyright 1977 West Publishing Co. ships among persons which may include others as well as self (Jones and Young, 1971). It may also be more specifically regarded as the structure which allows the formulation of persons and their actions in terms that are strictly psychological (Heider, 1958; Taguiri \& Petrullo, 1958).

Models of the individual's cognitive structure frequently afford systematic and parsimonious approaches to person perception, and the notion of an underlying structure which combines and differentiates person information has remained basic to the investigation of person perception. Asch (1946) investigated the manner in which specific traits are combined to form a total impression of a person. His investigation seemed to suggest a dynamic function rather than a simple additive one. Bruner and Taguiri (1954) sought to encourage the investigation of the rules of impression formation which represented the bases of "lay personality theories" used by people when interacting with others. Hastorf, Richardson, and Dornbusch (1958) specified three aspects of perception as important in the study of interpersonal perception: 1) ". . . the qualities of experience in social perception." Qualities here correspond to categories or dimensions one "employs in talking about other people." 2) "... the determinants of these specific experiences." 
These would be social psychological variables which correlate with experienced differences among stimulus persons. 3) ". . . the consequences of a specific perception." That is, what behaviors can be predicted from given sets of categories or dimensions and determinants. Hastorf et al. cite the need to study the perceptual categories actually used and their interrelationships with other aspects of social behavior of the perceiver. They urge that too much attention has been paid to the mere accuracy of social perceptions and that our understanding of relationships has benefitted little from accuracy investigations.

The personal construct psychology of George Kelly (1955) emphasized the individuality of a person's system of construing events. The saliency of a construct dimension in a person's system is determined by its utility in anticipating behavior or events and thus has a direct association with behavior prediction.

The application of multidimensional scaling (MDS) to social perception intensified the concern for the description of dimensions and their functional relationships among themselves as well as with behavior (Jackson, Messick, and Solley, 1957). MDS offers clear structural models for the combination of information in predicting behavioral responses, thus allowing an explicit investigation of the kinds and usefulness of various combination functions. The definitions of the models in terms of geometric rules of Euclidean algebra avoids the ambiguity of less rigorous and specifiable models such as Asch's (1964).

There are at least two categories of problems which have beset the measurement of the various relations which have been hypothesized to exist in the domain of person perception. The first, and by far the largest problem, is the frequent case in which the measurement process results in too few parameters to either adequately test a multi-faceted hypothesis or scale variables given a multi-component system of relations among those variables (Cronbach and Gleser, 1953; Cronbach, 1955, 1958; Jackson,
1962). The second, lesser problem involves the restrictions and limitations placed on the possible responses a subject may make as well as the stimuli the subject is responding to (Rosenberg and Sedlak, 1972; Kuusinen and Nystedt, 1972).

A usual case involving the first problem is the study in which a heterogeneous set of items is administered and the responses are combined into a single global index of a person's perception of, say, empathy in another or himself. This procedure ignores the various possible item content dimensions in addition to their relations to the criterion.

Perhaps this general criticism of too few parameters can also be applied to those traditional sociometric methods for assessing social structure which result in "sociograms" (Bferstedt, 1956; Moreno, 1951). These involve asking each individual in a group to choose, usually according to some affective criterion (liking, for example), one or more other members of the group. The group structure is then represented, nonparametrically, by a system of circles, for individuals, and arrows, for choices. The "geometry" of such a space has meaning only interpretively and has no mathematical properties which could be related to any other criteria. Kelly's (1955) Repertory Grid Test (REP Test) also represents a popular method which supplies too few parameters for the investigation of specific questions concerning the relationship between stimuli and constructs.

The second problem involves two general types of research design, inferential and descriptive. The inferential design frequently involves personality trait names. The investigation typically presents the subject with a personality trait name (or names) as descriptive of some individual and then asks the subject to make inferences about the presence of other traits in the individual. For example, the inference may be in terms of the likelihood of the presence of some other trait in the individual. Various psychometric procedures such as factor analysis (Peabody, 1967; Rosenberg and Olsham, 1970) and multidimensional scaling (Hays, 1958; Landis and 
Slivka, 1970) have been used to extract structures from inference data.

The descriptive design incorporates various methodologies. Trait-sorting involves the assignment of trait names from a given set to specified individuals under various sets of rules (Rosenberg, Nelson and Vivekananthan, 1968). Checklists are a special case of trait-sorting and have long been in use (Thurstone, 1934; Cliff, 1968).

The above descriptive and inferential methodologies all have the property of restricting the subject to the use of a preselected vocabulary or set of scales. Relative to the semantic differential (SD) type design, Cliff (1973) cites two disadvantages. The first is that "any stimulus dimension not represented in the scales will not emerge from the semantic differential analysis even though it is important for differentiating stimu1i." The second involves the consideration that any dimension which is recovered, is "weighted by the number of scales representing (it) as well as by (its) salience to the judge." MDS procedures which do not involve restrictions on the dimensions of judgment, as in a paired comparisons task, are not subject to these two criticisms. Even in this case, however, the identification of resulting dimensions may still be subject to the problems of a preselected vocabulary unless adequate care is taken in the selection of the vocabulary.

There are various reasonable solutions to this identification problem. Perhaps the least restrictive are "free-response" methods (Kelly, 1955; Hastorf, Richardson and Dornbusch, 1958; Beach and Wertheimer, 1961; Rosenberg and Sedlak, 1972). These methods allow the subject to describe individuals, differences among individuals, and groups in his own words. As Cronbach (1958) implies, these are most appropriate for individual diagnostic uses but result in a cumbersome task for the experimenter if he wishes to make comparisons across individuals or groups.

The present research investigates the application of free-response data in conjunction with MDS for representing an individual's cognitive structure underlying person perception.
Unfortunately, traditional MDS methods require a prohibitively large number of responses from an individual in order to scale only a moderately large number of stimuli (e.g. 300 responses for 25 stimuli). In response to this problem, Young \& Cliff (1972) presented and tested a computer interactive MDS procedure, Interactive Scaling with Individual Subjects (ISIS). This program greatly reduced the number of necessary responses an individual needed to make for large sets of stimuli. The present investigation uses another computer-interactive procedure, INTERSCAL (Cliff, Green, Kehoe, and Doherty; 1976, in press), which is a second generation refinement of ISIS.

Briefly, INTERSCAL works as follows: 1) A subject, seated in front of a computer terminal, judges how dissimilar the elements of a pair of stimuli are as INTERSCAL presents the pairs on the terminal; 2) After a small number of initial responses, INTERSCAL determines a set of $r+1$ basis stimuli which appear as eutliers in an estimated $r$-dimensional space; 3) Once selected, the basis stimuli are paired with new stimuli and the process continues until all stimuli have been paired with the final set of basis stimuli; 4) INTERSCAL then produces a multidimensional map of the stimuli for the individual subject with stimulus values on the separate dimensions and various other evaluative statistics. More specifically, the output contains goodness-of-fit indices between the actual dissimilarity judgments and distances, calculated from the recovered multidimensional structure. Also included in the output is a measure of the subject's reliability and the predictive validity of the recovered structure.

\section{The Model}

Most MDS methods, including INTERSCAL, incorporate a general model in which the structure for a given domain of stimuli can be represented in simple, geometric terms. Given, say, a two-dimensional space or domain, one stimulus is distinct from another insofar as they differ on the two dimensions. In geometric terms, the 
stimuli lie at different points in the space and these points are determined by the corresponding stimulus values on the two axes. Furthermore, the overall distance between any two points in a geometric space can be easily determined by knowing the axis values of the two points in question. The difference between two stimuli in psychological terms, then, is represented as the distance between them in geometric space and depends upon their individual qualities with respect to the salient dimensions of meaning. Thus, MDS determines dimensional structures in a way that: 1) allows the psychological salience of underlying dimensions to have primary influence on measured salience; 2 ) defines components of observed judgments; and 3) defines a simple and informative map-like representation of structural properties.

The present research was undertaken with the following three objectives: 1) to examine the utility of INTERSCAL for the representation of individual role structures; 2) to investigate the congruence of the REP Test, INTERSCAL, and SD methods for the measurement of rule structure; and 3) to investigate the consistency of role structure over a period of ten weeks.

\section{Method}

\section{Subjects}

Subjects were 27 introductory psychology students who fulfilled credit requirements for the class and four volunteer high school students.

\section{Tasks and Stimuli}

Stimuli consisted of 23 role descriptions taken from Kelley's (1955) REP Test (see Table 1). Each student supplied the names of persons who corresponded to the role description at the time of each of two sessions. In the two INTERSCAL sessions, these 23 different names were entered into the computer by the experimenter and served as the stimuli for each student. Role descriptions were constant for each student, but, obviously, the actual stimulus-persons differed. The role descriptions were chosen so as to in- clude a set of persons which more or less spanned each student's "social space".

Each student had four separate tasks to perform during the course of the investigation. Each task utilized the same 23 role descriptions but the stimulus-persons supplied by the student were not necessarily exactly the same from task to task. Students were asked to supply names of persons who were appropriate at the time of the particular task. At the beginning of the fall semester each student completed an INTERSCAL session and, within 10 days, a paper-andpencil version of the REP Test. Half of the students completed the REP Test before the INTERSCAL session and the order was reversed for the other half. At the first INTERSCAL session, immediately after the names of the role persons for that subject were entered, the computer began to present the selected pairs to the student on a CRT terminal screen. For each pair, one at a time, the student typed back to the computer a number from 1 to 9 . Low numbers indicated that the two stimulus-persons were judged to be similar ( 1 for most similar) while higher numbers indicated dissimilarity $(9$ for most dissimilar). During the session, 27 pairs were presented twice and the repeated judgments were used to estimate reliability. At the end of the INTERSCAL session, students were asked to describe in their own words the differences and obvious similarities between selected pairs of stimulus-persons which were apparent outliers in their MDS solution. Students' descriptions corresponding to the ends or poles of the axes were used both to name and to construct ten SD scales used in subsequent stages of the research.

The REP Test (Kelly, 1955) requires each person to decide which two of three stimulus-persons are similar and yet differ from the third according to the characteristic on which they are similar. The version of the REP Test used included 27 triads and each student described the difference and similarity that determined their judgment for each triad.

Ten weeks later, at the end of the fall semester, each student again completed the INTER- 
TABLE 1

\section{Role Descriptions}
1)
Self
2) Your mother. (Or the person who has played the part of a mother in your life.)
3) Your father. (Or the person who has played the part of a
4) father in your life.)
5) The person in whose presence you feel the most threatened.
6) A teacher you disliked. (Or the teacher of a subject you dislike.) A teacher you liked. (Or the teacher of a subject you liked.)
7) Your closest present friend of the same sex.
8) Your wife (or husband) or closest present girl (boy) friend.
9) The person whom you would most like to be of help to, or whom you feel most sorry for.
10) Your brother nearest your
11) who is most like one.) Your sister nearest you
12) toward. who is most like one.)
A once close friend of the same sex whom you now feel negatively
13) The person whom you have met in the past six months whom you would
14) like to know better.
14) The most happy person that you know personally.
15) Your closest girl (boy) friend immediately before you started
16) going with your wife (husband) or present closest (girl, boy) friend.
17) A nelghbor with whom you get along well.
18) The most interesting person whom you know personally. The most ethical person that you know personally.
19) An employer, supervisor, or officlal under whom you worked and whom you found hard to get along with.
20) The most successful person whom you know personally.
21) The most intelligent person, in your own age group, whom you
22) know personally.
23) A nelghbor whom you find hard to understand. A person with whom you have been closely associated, who for some unexplained reason, appeared to dislike you. 
SCAL procedure with the same role descriptions, although the persons in some of the roles may have differed. Within ten days following the second INTERSCAL session, each $S$ also rated each of 23 stimulus-persons on ten different SD scales (Table 2). These scales were derived by classifying the descriptions of differences and similarities between stimulus pairs obtained at the end of the first INTERSCAL session. These scale ratings were subsequently used to aid in the identification and interpretation of the psychological properties of the recovered structures.

Thus, there were four sessions. At each session the students provided names of 23 standard role persons. In the two INTERSCAL sessions students made dissimiliarity judgments of the

TABLE 2

DESCRIPTIVE SCALES

SCALE VALUES

SCALES

1)

\begin{tabular}{ll}
\hline HIGH STATUS & LOW STATUS \\
IMPORTANT & UNIMPORTANT \\
PROMINENT & NOT PROMINENT \\
\hline COLD & WARM \\
CRUEL & KIND \\
RUTHLESS & \\
\hline
\end{tabular}

3)

\begin{tabular}{lll} 
OLD YOUNG & Y \\
\hline
\end{tabular}

4)

\section{QUIET}

LOUD

RESERVED BRASH

GENTLE UNCOUTH

$\begin{array}{ll}\text { POLITE BOORISH } & \text { BOE }\end{array}$

$\begin{array}{ll}\text { 5) LACKING IN SELF CONFIDENCE } & \text { SELF-CONFID } \\ \text { DEPENDENT } & \text { INDEPENDENT } \\ \text { IMMATURE } & \text { MATURE }\end{array}$

IMMATURE MATURE

6)

$\begin{array}{ll}\text { FRIENDLY UNFRIENDLY } & \text { UNOF }\end{array}$

6) AMIABLE NOT AMIABLE
ACCPIIMG

$\begin{array}{ll}\text { ACCEPTING REJECTING } & \end{array}$

$\begin{array}{ll}\text { INTELLIGENT } & \text { UNINTELIIGENT }\end{array}$

7) AWARE UNAWARE

SMART DULL

THOUGHTFUL

UNCONCERNED

CONCERNED

LACKS UNDERSTANDING UNDERSTANDING

$\begin{array}{ll}\text { LACKS FEELING EMPATHETIC } & \text { EMP }\end{array}$

$\begin{array}{ll}\text { DOMINEERING } & \text { NOT DOMINEERING } \\ \text { DOMINATING } & \text { NOT OPRRESSIVE }\end{array}$

$\begin{array}{ll}\text { NOT OPPRESSIVE } & \text { NOTINATING }\end{array}$

PATIENT IMPATIENT

REASONABLE UNREASONABLE

EVEN-TEMPERED HOT-TEMPERED

EVEN TEMPERED I


role persons. In the REP Test students made triadic judgments. In the last session students rated the role persons on ten SD scales derived from the free-response data of the first session.

\section{Analyses}

The structures defined by INTERSCAL are related to two kinds of dissimilarity judgments. The first kind are those dissimilarity judgments which INTERSCAL used to derive the structures. The correlation between these 'used' judgments and the corresponding distances in a derived structure constitutes a goodness-of-fit index for that structure. The second kind are those 27 repeated dissimilarity judgments which were collected but not used by INTERSCAL to derive the structures. The correlation between these 'unused' judgments and the corresponding distances in a derived structure constitutes an index of predictive validity for that structure. These indices were computed for each student.

A combination of free-responses interpretation (e.g. Beach \& Wertheimer, 1961; Hastorf, Richardson \& Dornbusch, 1958; Rosenberg \& Sedlak, 1972) and traditional correlational methods of interpretation was used to interpret the psychological dimensions of each student's structure. At the end of the first INTERSCAL session each student described in his own words the salient differences and similarities among the outlying "basis" stimulus-persons. These descriptions from all 31 Ss were sorted and grouped into ten relatively distinct and frequently occuring trait categories in a manner similar to that of Cattell (1943). Key words identifying the poles of each trait category were used to define the ten SD rating scales.

The interpretation analyses consisted of correlating the stimulus-persons' ratings on a particular SD scale with their axis values on the structural dimensions derived by INTERSCAL. The degree to which the INTERSCAL dimensions correlate with an SD scale defines a $d^{-}$rection in the INTERSCAL structure which corresponds to the meaning of that particular $S D$ scale. For example, if the first dimension corre- lated .90 with the "age" scale and the second dimension correlates .05 , then the first dimension could be meaningfully interpreted as an "age" dimension. That is, the age of stimulus-persons is an apparently important discriminator for that student and corresponds closely to a recovered structural dimension. Of course, trait scale directions are not required to fall along one or another recovered structural dimension. If, for example, the "age" scale correlations with the two dimensions were 6 and .6 , the "age" direction would run diagonally between the two structural dimensions, rather than close to either. Once all of the psychologically meaningful trait directions are located in the recovered structure, the structural dimensions could be rotated so as to correspond to particular trait directions. This rotation allows the axis-values of the stimuli to be defined on psychologically meaningful dimensions.

The congruence of the REP Test method and the INTERSCAL structure for each student was assessed by using the INTERSCAL structure to predict the student's REP Test triad responses. For each triad in the REP Test the prediction is that the stimulus-persons closest together in the INTERSCAL structure are the two judged as similar in the REP Test triad. The remaining two pairs are farther apart in the INTERSCAL structure and are predicted to be judged as dissimilar in the REP Test triad. For each student a biserial correlation was computed between the interpoint distances in the INTERSCAL structure and the similar-dissimilar REP Test dichotomy.

\section{Results}

\section{The Judgments}

Table 3 summarizes information about the judgments. It can be seen that the average num. ber of judgments a student was required to make, excluding the repeated judgments, was about 154 for the first session and 156 for the second session, considerably fewer than the 253 pair-wise judgments of 23 stimuli which MDS 
procedures traditionally require. This savings averages nearly $40 \%$ and could be expected to increase as the number of stimuli increases. This reduced number of judgments is a basic advantage of INTERSCAL.

The mean number of judgments reported in Table 3 does not include the 27 repeated judgments each student made at various times during an INTERSCAL session. These 27 judgments served to determine an estimate of re- liability of the judgment task for each student. The reliability index is the correlation between the scale values of the first and second set of judgments of the same 27 pairs.

These reliabilities ranged from moderate to very high in both sessions; the median for both was .85 . The judgment task itself is apparently sufficiently reliable to warrant serious consideration of consistent underlying structures for this set of stimuli. In a monte carlo investigation

TABLE 3

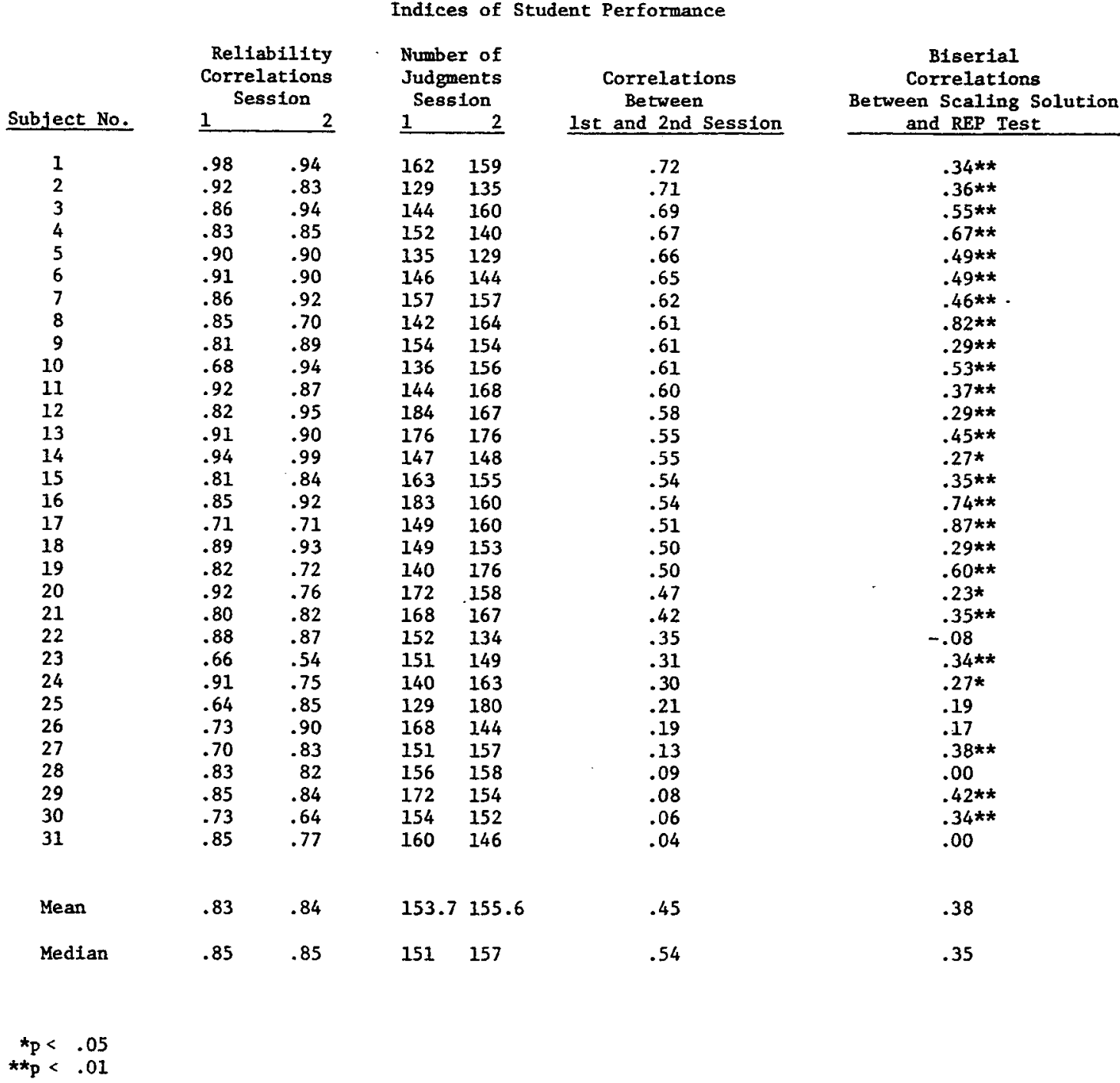


Girard (1973) found that at low and moderate error levels INTERSCAL recovered true structures appreciably better than a non-interactive cyclic design for 50 stimuli with a deletion of $67 \%$ of the pairs. This was not the case at the highest error level. The reliability of the present data represents in Girard's study a moderately high error level at which INTERSCAL's advantage over the non-interactive cyclic design is somewhat reduced. The differences in number of stimuli and reduction percentages obscure the meaningfulness of Girard's study for this particular application. Indeed, as the reduction percentage decreases the differences between interactive and non-interactive methods would be reduced.

\section{Interpretation of Structures}

The interpretation of structures involves two related steps: 1) an evaluation of the appropriate dimensionality; and 2) the correlation of the retained dimensions with each of the ten SD scales individually. First, dimensionality may be assessed for each student by the goodness-of-fit and predictive validity indices. The average goodness-of-fit correlations for structures of one, two, three, and four dimensions were .62 , $.76, .84$, and .88 respectively. These averages represent the typical result that two- or three-dimensional solutions are most appropriate for students according to the goodness-of-fit criterion. On the other hand, the average predictive validities for the four solutions were $.60, .65, .67$, and .66 , respectively. According to the predictive validities one or two dimensions are typically the appropriate dimensionality. For the sake of comparability and in order to further evaluate dimensionality, the correlations of the SD scales with the two-dimensional solutions for each student were examined.

Results for two particular students, which are typical of those obtained from nearly all students, are shown in Figures 1 and 2 where " $a$ " indicates first session structures and " $b$ ", second session. The axes labeled $I$ and $I I$ in each structure have been rotated to correspond close- ly to the trait directions in each structure.

The similarities between $S 9$ and $S 19$ are quite typical of nearly all students. Looking at these trait directions, a general and consistent trend emerges. The trait-scales "cold" and "thoughtless" are usually clustered together at one end of a single trait dimension. At the other end of the same dimension one usually finds the traitscales "friendly", "patient" and "quiet". These scales reflect a pervasive evaluative dimension similar to those consistently recovered in the scaling of personality trait spaces. The remaining trait-scales, "important" and "intelligent", are frequently near or in the "friendly" cluster, tending to increase the generality of the evaluative trait dimension.

The primary difference between $S 9$ and $S 19$ is in the degree to which a second direction or dimension of meaning is indicated by the traitscale correlations. Specifically, there does not seem to be a psychologically meaningful second trait direction for S9. However, for S19, the "age" and "dominance" trait-scales seem to indicate a meaningful second direction that is quite different from the first dimension direction with which "age" and "dominance" do not correlate at all. For other subjects a second trait dimension, if defined at all, is defined by some combination of the trait-scales "age", "dominance" and "maturity" as for S19. These suggest a trait-dimension which corresponds to Osgood's "potency" dimension in semantic spaces. Establishment of such a correspondence, of course, would require more specific scales. This difference between $S 9$ and $S 19$ is observable on the structures from both the first and second INTERSCAL session structures. That is, the differences between the first session structures in Figures $1 \mathrm{a}$ and $2 \mathrm{a}$ are very much like the differences between the second session structures in Figures $1 \mathrm{~b}$ and $2 \mathrm{~b}$. This between-session consistency of trait directions is typical of virtually all students.

\section{Changes over Time}

Of particular interest may be the changes over 
SUBJECT 9

First session INTERSCAI structure

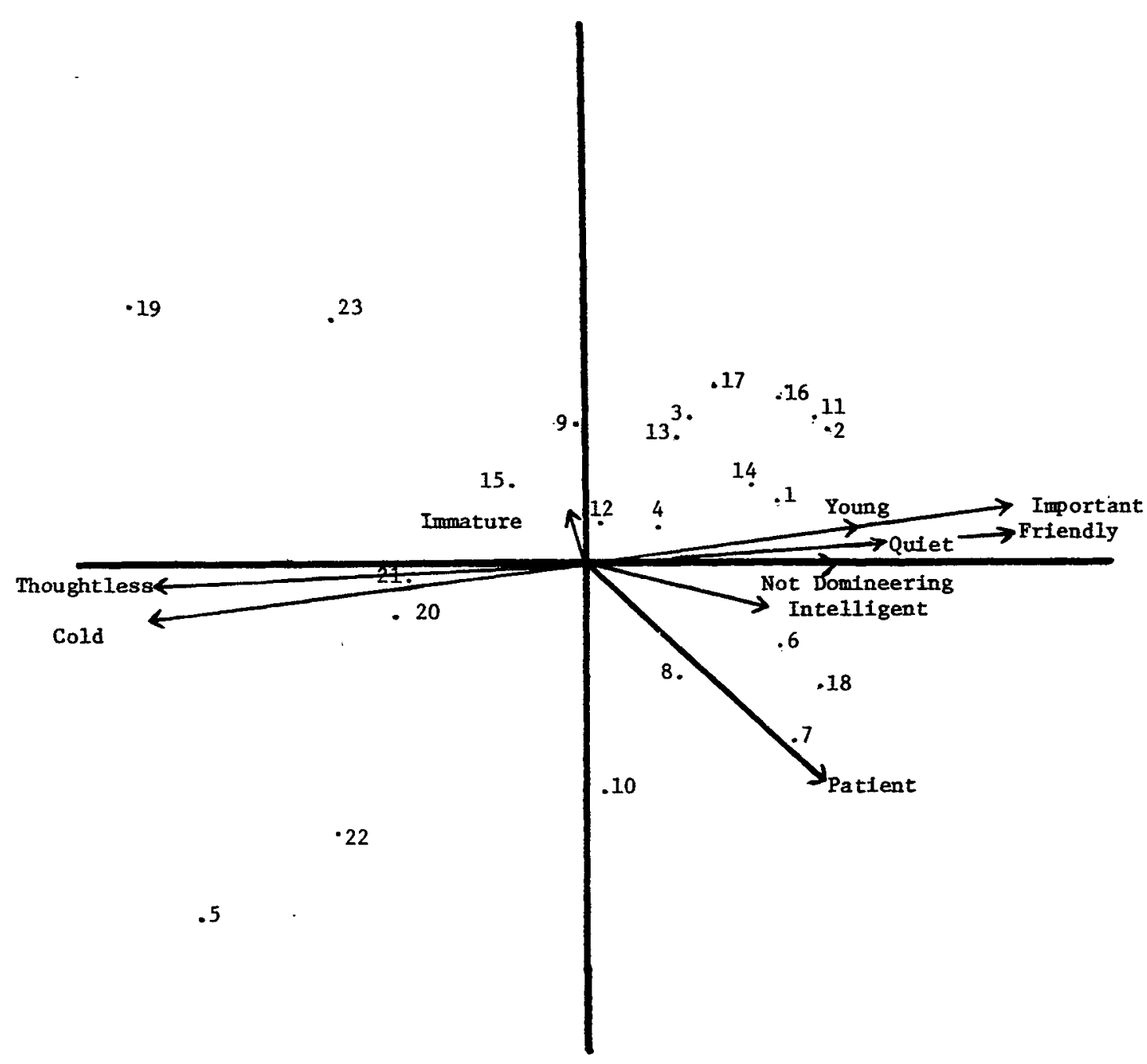

Flgure la

time of the relative locations of the stimulus-persons. This was studied by an analysis of the distances between stimulus-persons in the recovered structures. The 253 distances derived from a student's first session structure were correlated with those derived from the second session structures. Table 3 presents this inter-session correlation for each student. As can be seen, the median correlation is .54 with a few very low values. It is apparent, though, that most of the students maintained a statistically significant degree of structural consistency over the course of about ten weeks. S9 and S19 had intersession correlations of .61 and .50 , respectively. Thus, the impression of trait dimension consistency is complemented by the consistency of 


\section{SUBJECT 9}

Second session INTERSCAL structure

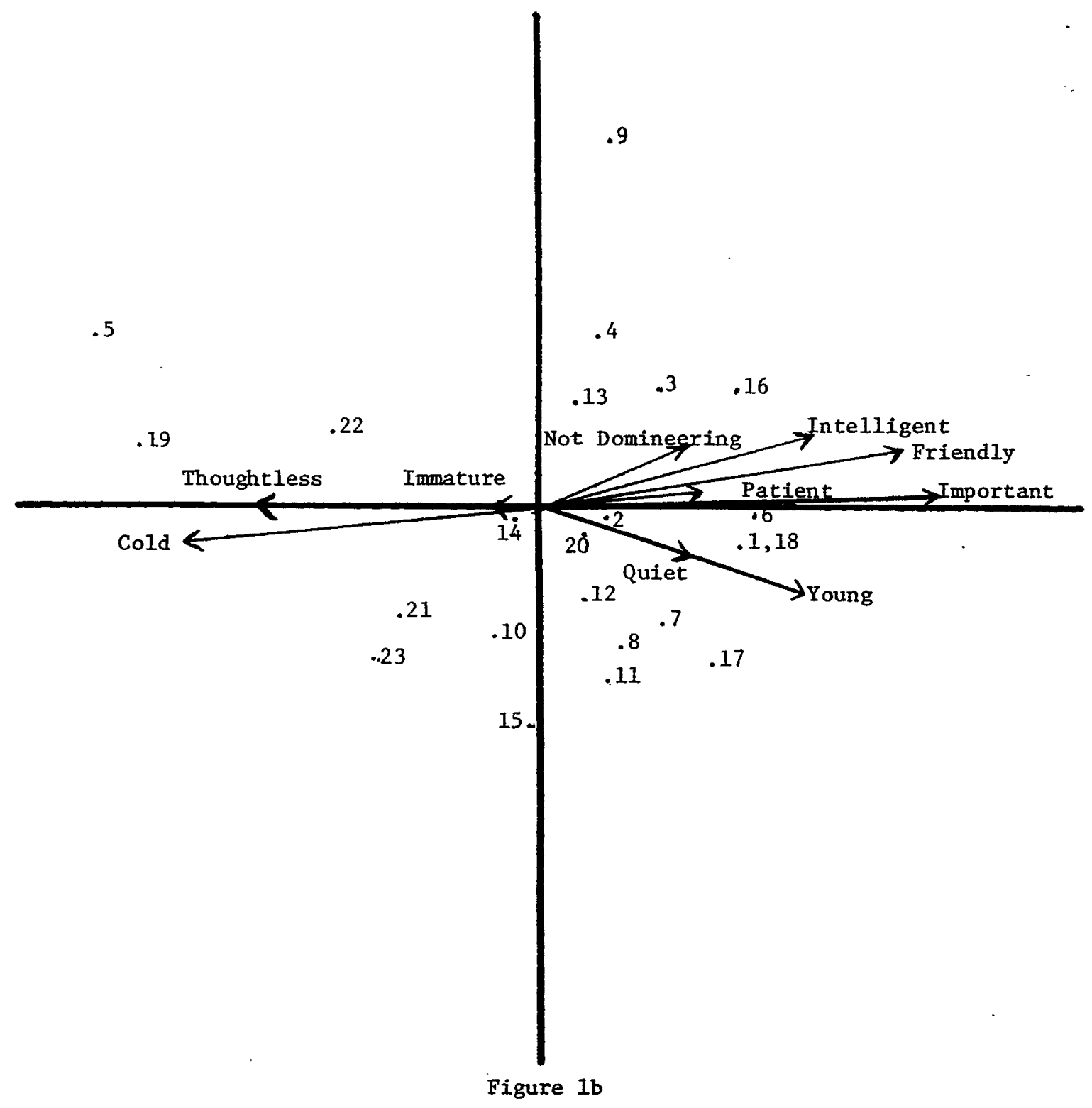

relative location. Such longitudinal consistencies indicate that the structures merit serious attention in terms of identifying a student's salient psychological characteristics. Moreover, such overall consistency suggests that any substantial change in the position of one role relative to the others may reflect important changes in a student's perception of that role. 


\section{Relationship between INTERSCAL and REP Results}

One approach to the evaluation of INTERSCAL involves the generalizability of the obtained structures. A further validation of the structural model results if the INTERSCAL structures obtained from overall dissimilarity judgments could predict judgments of a somewhat different kind gathered at a different time. The REP Test provided such judgments. Recall that it was administered within ten days of the first INTERSCAL session. As described above, the REP Test judgments were predicted by the

SUBJECT 19

First session INTERSCAL structure

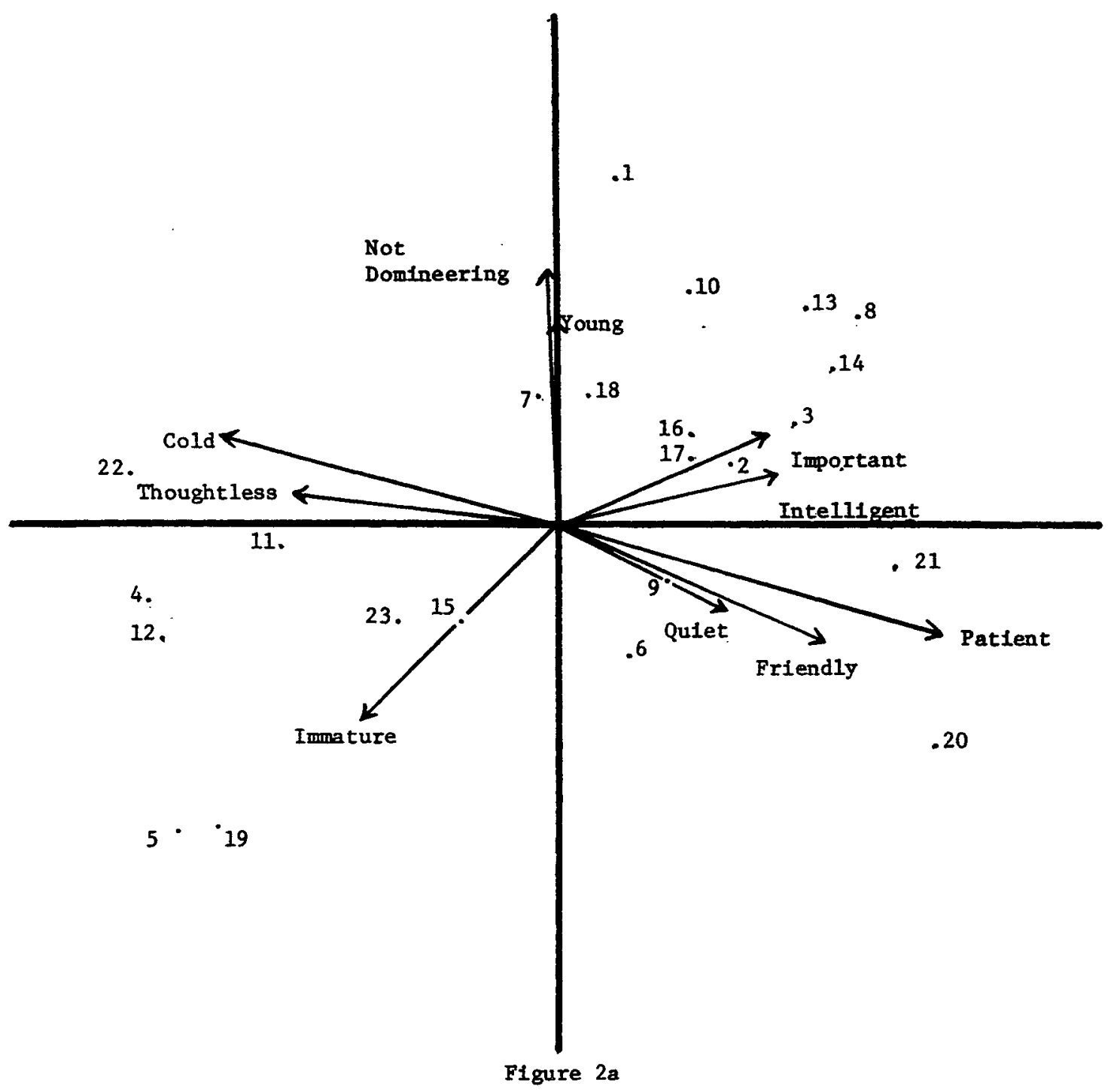


INTERSCAL structures. For each $S$, then, there is a biserial correlation between the INTERSCAL prediction of REP Test judgments and actual REP Test judgments. Table 3 presents these correlations. All but five were statistically significant $(\mathrm{p}<.05)$, and the median correlation was .35 . These correlations gain additional in- tuitive significance when one considers that all INTERSCAL predictions were made using only two recovered dimensions while each of the 27 REP Test triads could easily result in the use of a different pervasive or unique dimension. Each triad of the REP Test is intended to generate a single construct or dimension. While it is as-

SUBJECT 19

Second session INTERSCAL structure

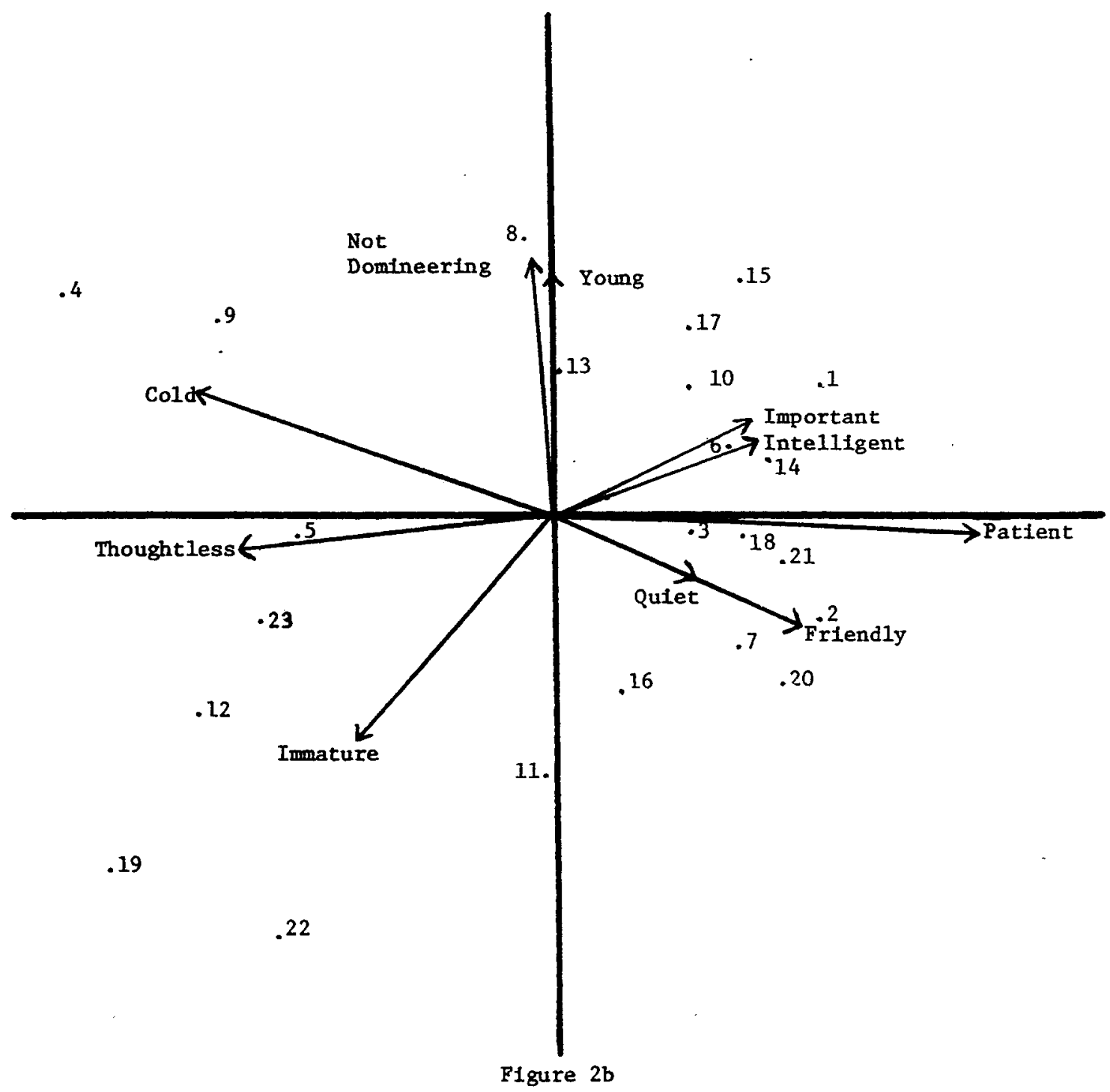


sumed that important and pervasive dimensions have to do with both pair comparison dissimilarity judgments and REP Test triadic judgments, it is also likely that the restrictions placed on the selection of a construct in characterizing a triad would tend to generate more idiosyncratic dimensions than for the less constrained pair comparison judgments. Thus the psychological importance of the two retained INTERSCAL dimensions is reinforced to the extent that the two dimensions account for triadic judgments which may facilitate the use of idiosyncratic dimensions.

\section{Discussion}

The INTERSCAL method defined consistent structural dimensions which were pervasive and general, accounting for: 1) the dissimilarity judgments from which the dimensions were derived; 2) other independent dissimilarity judgments; 3) REP test judgments; and 4) trait-scale rating judgments. Further, these interrelationships of structure and judgments were achieved with structures determined from nearly $40 \%$ fewer judgments than usually required in MDS.

The MDS model exemplified by INTERSCAL frequently affords a simple geometric representation of an individual's perception. In turn, this allows a psychologically meaningful characterization of the objects of perception. Such derived cognitive structures, with their straightforward mathematical properties, allow for definitions of similarity among the structures of several individuals.

The ability to make such individual analyses, of course, opens the way to another application of INTERSCAL. Initial assessment of psychotherapy and changes during therapy might be supplemented by use of the MDS procedure. For example, an operational definition of empathy or accuracy of clinical impressions might be the dimensional similarity of a clinician assuming the client's identity compared to the actual perceptual structures of the client. Group therapy situations provide another setting for application. A clinician's impressions of individual members' similarities and sociometric status can be related to the actual individual differences and friendship choices.

For such applied uses, the manner in which a few individual structures may be most beneficially compared may well be somewhat removed from the typical psychometric approaches to the evaluation of individual differences. For example, the relative location and changes in the relative location of particular stimulus-points may be of considerable interest to a counselor or therapist. These particular kinds of information, however, are matters of careful visual inspection rather than, or in addition to, more wholistic measures of structural similarity such as dimension-wise correlations and axis-wise goodness-of-fit indices.

The value of visual inspection is, of course, dependent upon the reliability and generalizability of the method. The present research demonstrates sufficient judgment reliability, structural fit and predictive validity, and methodological generalizability for INTERSCAL, in low dimensional domains, that any substantial change of one stimulus-point relative to the others may well reflect important changes in the perception of that stimulus-point. Thus, interactive MDS procedures such as INTERSCAL appear to have a variety of psychological applications.

\section{References}

Asch, S. E. Forming impression of personality. Journal of Abnormal and Social Psychology, 1946, 41, 258-290.

Beach, L., and Wertheimer, M. A free-response approach to the study of person cognition. Journal of Abnormal and Social Psychology, 1961, 62, 367374.

Bferstedt, A. The methodology of preferential sociometry. Sociometry Monograph, 1956, No. 37.

Bruner, J. S. and Taguiri, R. Person preception. In G. Lindzey (Ed.), Handbook of Social Psychology. Reading, Mass.: Addison-Wesley, 1954, Vol. 2. pp. 634-654.

Cattell, R. B. The description of personality: Basic traits resolved into clusters. Journal of Abnormal and Social Psychology, 1943, 38, 476-506.

Cliff, $\mathrm{N}$. Adjective check list responses and individual differences in perceived meaning. Educational Psychological Measurement, 1968, 28, 1063-1077.

Cliff, N. Scaling. Annual Review of Psychology, 1973 
Cliff, N., Green, R., Kehoe, J., and Doherty, L. M. INTERSCAL: A TSO FORTRAN IV Program for Subject Computer Interactive Multidimensional Scaling. Educational and Psychological Measurement, 1976, 35, in press.

Cronbach, L. J. Processes affecting scores on "Understanding of Others" and "Assumed Similarity". Psychological Bulletin. 1955, 52, 177-192.

Cronbach, L. J. Proposals leading to analytic treatment of social perception scores. In R. Taguiri and L. Petrullo (Eds.), Person Perception and Interpersonal Behavior, 1958, 352-379.

Cronbach, L. J. and Gleser, G. C. Assessing similarity between profiles. Psychological Bulletin, 1953, 50. $456-473$

Girard, R. A. A monte carlo evaluation of interactive multidimensional scaling. Unpublished doctoral dissertation, University of Southern California, Los Angeles, 1973.

Hastorf, A. H., Richardson, S. A., and Dornbusch, S. $M$. The problem of relevance in the study of person cognition. In $R$. Tagiuri and $L$. Petrullo (Eds.), Person Perception and Interpersonal Behavior. Stanford, California: Stanford University Press, 1958.

Heider, I. Perceiving the other person. In R. Taguiri and L. Petrullo (Eds.), Person Perception and Interpersonal Behavior. Stanford: Stanford U. Press, 1958, pp. 22-26.

Jackson, D. N. The measurement of perceived personality trait relationships. In N. F. Washburne (Ed.), Decisions, Values and groups. Oxford: Pergamon Press, 1962.

Jackson, D. N., Messick, S., and Solley, C. M. A multidimensional scaling approach to the perception of personality. Journal of Psychology, 1957, 44, 311-318.

Jones, L. E. \& Young, F. W. Structure of a social environment: Longitudinal individual differences scaling of an intact group. Journal of Personality and Social Psychology, 1972, 24, 108-121.

Kelly, B. A. A Theory of Personality: The Psychology of Personal Constructs. New York: Norton, 1955.

Kuuisinen, J. \& Nystedt, L. Individual vs. provided constructs cognitive complexity and extremity of ratings in person perception. Report from the Psychological Laboratories U. of Stockholm, 1972.
Landis, D. \& Slivka, R. M. Some determinants of multidimensional points of views: Two studies. Perceptual and Motor Skills, 1970, 31, 659-670.

Moreno, J. L. Sociometry Experimental Method and the Science of Society. New York: Beacon House, 1951.

Osgood, C. E., Suci, G. J., and Tannenbaum, P. H. The Measurement of Meaning. Urbana: University of Illinois Press, 1957.

Peabody, D. Trait inferences. Evaluative and descriptive aspects. Journal of Personality and Social Psychology, 1967, 7 (4, whole No. 644).

Rosenburg, S., Nelson, C. and Vivekananthon, P. S., A multidimensional approach to the structure of personality impression. Journal of Personality and Social Psychology, 1968, 9. 283-294.

Robenburg, S. and Olsham, K. Evaluative and descriptive aspects in personality perception. Journal of Personality and Social Psychology. $1970,16.619-626$.

Rosenburg, S. and Sedlak, A. Structural representation of perceived personality trait relationships. In A. K. Romney, R. N. Shepard, and S. B. Nerlove (Eds.), Multidimensional Scaling: Theory and Applications in the Behavioral Sciences. Vol. II, New York: Seminar Press, 1972.

Taguiri, R. T., Petrullo, L. (Eds.), Person Perception and Interpersonal Behavior. Stanford: Stanford U. Press, 1958, pp ix-rvii.

Thurstone, L. L. The vectors of mind. Psychological Review, 1934, 41, 1-32.

Young, F. W. and Cliff, N. Interactive scaling with individual subjects. Psychometrika, 1972, 37, 385415.

\section{Acknowledgements}

The present investigation was supported in part by Public Health Service Grant MH 16474. The authors wish to thank Norman Cliff. William McGarvey, and Jody Gurrola for their assistance in the preparation of this manuscript.

\section{Author's Address}

Jerard F. Kehoe, Department of Psychology, Virginia Polytechnic Institute and State University, Blacksburg, VA 24061 